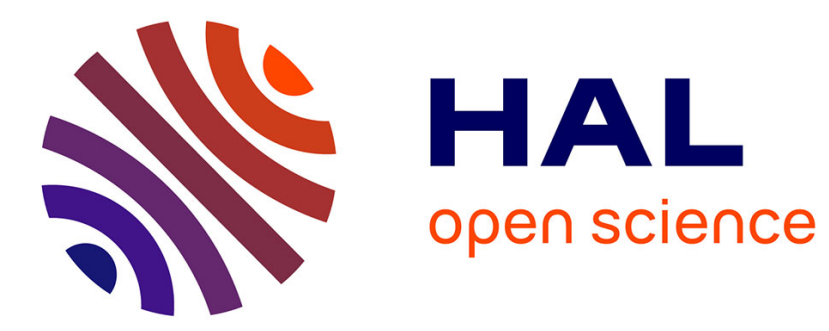

\title{
La légitimité précaire de l'expertise. Un " Centre de ressources " entre science et politique : le Centre Alain Savary (1993-2004) \\ Philippe Bongrand
}

\section{- To cite this version:}

Philippe Bongrand. La légitimité précaire de l'expertise. Un " Centre de ressources " entre science et politique : le Centre Alain Savary (1993-2004). Carrefours de l'éducation, 2014, 37 (1), pp.15-27. 10.3917/cdle.037.0015 . hal-03184335

\section{HAL Id: hal-03184335 \\ https://hal.science/hal-03184335}

Submitted on 1 Apr 2021

HAL is a multi-disciplinary open access archive for the deposit and dissemination of scientific research documents, whether they are published or not. The documents may come from teaching and research institutions in France or abroad, or from public or private research centers.
L'archive ouverte pluridisciplinaire HAL, est destinée au dépôt et à la diffusion de documents scientifiques de niveau recherche, publiés ou non, émanant des établissements d'enseignement et de recherche français ou étrangers, des laboratoires publics ou privés. 


\section{LA LÉGITIMITÉ PRÉCAIRE DE L'EXPERTISE. UN « CENTRE DE RESSOURCES » ENTRE SCIENCE ET POLITIQUE : LE CENTRE ALAIN SAVARY (1993-2004)}

\section{Philippe Bongrand}

Armand Colin | « Carrefours de l'éducation »

2014/1 $n^{\circ} 37$ | pages 15 à 27

ISSN 1262-3490

ISBN 9782200928896

Article disponible en ligne à l'adresse :

https://www.cairn.info/revue-carrefours-de-l-education-2014-1-page-15.htm

Distribution électronique Cairn.info pour Armand Colin.

(C) Armand Colin. Tous droits réservés pour tous pays.

La reproduction ou représentation de cet article, notamment par photocopie, n'est autorisée que dans les limites des conditions générales d'utilisation du site ou, le cas échéant, des conditions générales de la licence souscrite par votre établissement. Toute autre reproduction ou représentation, en tout ou partie, sous quelque forme et de quelque manière que ce soit, est interdite sauf accord préalable et écrit de l'éditeur, en dehors des cas prévus par la législation en vigueur en France. Il est précisé que son stockage dans une base de données est également interdit. 


\section{Philippe Bongrand}

philippe.bongrand@u-cergy.fr

\section{LA LÉGITIMITÉ PRÉCAIRE \\ DE L'EXPERTISE. UN «CENTRE DE}

RESSOURCES » ENTRE SCIENCE

ET POLITIQUE : LE CENTRE

ALAIN SAVARY (1993-2004)

En 1981, pour lutter contre les inégalités sociales à l'école, le ministère français de l'Éducation nationale classe en zones d'éducation prioritaires (ZEP) des établissements scolaires situés dans des territoires ou « milieux sociaux où le taux d'échec scolaire est le plus élevé ${ }^{1}$. Initialement, aucune indication ne précise comment utiliser les crédits et personnels spécifiquement affectés: l'échelon central du ministère s'en remet aux acteurs de terrain, dont il s'agit de « libérer » les initiatives. Cette déconcentration administrative n'est pas remise en cause au cours de trente années d'existence, mais elle est nuancée par l'accumulation d'instruments de travail qui sont diffusés à l'échelle nationale : des « projets», « contrats », " partenariats », « évaluations » ou encore « expérimentations » qui, présentés comme des outils génériques ou prenant la forme d'exemples de mise en œuvre, viennent « accompagner » les professionnels de ZEP. Ces instruments, typiques de la « gouvernance » qui se déploie depuis les années 1980 dans les politiques publiques d'éducation (Dutercq, 2005 ; van Zanten, 2011) ou d'autres secteurs d'action publique (Pasquier, Simoulin \& Weisbein, 2007), sont de type procédural : ils engagent les « acteurs de terrain » à se saisir de méthodes tout en leur reconnaissant explicitement une marge de liberté pour définir les objectifs comme les modalités de leurs actions - ici pédagogiques.

1. Circulaire «Zones prioritaires », 1er juillet 1981, Bulletin officiel de l'Éducation nationale (BOEN), 9 juillet $1981, n^{\circ} 27$. 
Dans la mesure où ces instruments ne sont ni convergents, ni neutres (Lascoumes \& Le Galès, 2005), leur mobilisation dans l'action publique soulève un problème de légitimité: qui peut, à quel titre et en vertu de quels fondements les confectionner, les sélectionner ou inciter à leur utilisation ? Leur légitimité peut se fonder sur une logique de démocratie représentative (et top down), suivant laquelle un centre politique dirige l'action de terrain et impose ses orientations aux agents d'une mise en œuvre conçue comme principalement technique. Mais elle peut également reposer sur un argument pragmatique (et bottom up), mis en avant par les discours en faveur de la gouvernance, où ce sont les initiatives locales qui orientent l'action publique. Leur légitimité peut, enfin, être fondée sur les compétences (notamment professionnelles ou scientifiques) propres aux acteurs qui mettent ces ressources en circulation. Ces logiques ne sont cependant pas nécessairement exclusives, et leurs champs d'application respectifs ou leurs modalités d'articulation suscitent des hésitations ou tensions : comment, au moment de concevoir ou de mettre en œuvre leur rôle dans la politique des ZEP, les différents acteurs prennent-ils position?

Ces questions de légitimité se posent, entre autres, aux « centres de ressources » pour l'éducation prioritaire. Créés au sein du ministère de l'Éducation nationale au cours des années 1990, ces centres ont pour rôle d'« épauler »² les enseignants, responsables ou coordonnateurs de ZEP, en concevant, étudiant ou faisant connaître des exemples de mise en œuvre des instruments de la politique des ZEP. Parmi eux, le Centre Alain Savary affronte ces questions de légitimité de manière singulière. Créé en 1993 au sein d'un établissement public (I'Institut national de recherche pédagogique, INRP) relativement autonome des administrations « actives » élaborant et mettant en œuvre les politiques scolaires, le Centre Alain Savary ne se conçoit pas comme la courroie de transmission directe de décisions politiques, mais plutôt comme un lien entre une politique publique et le monde de la recherche. Ce centre ne rencontre donc pas seulement les incertitudes, évoquées plus haut, relatives aux modes de fonctionnement légitimes d'une politique publique déconcentrée: il conjugue ces incertitudes à celles relatives au rôle possible et souhaitable de la recherche dans la confection ou la circulation d'instruments pour la politique des ZEP - autrement dit, il met en cause la légitimité de l'expertise dans l'action publique (Delmas, 2011). Maillon de la circulation des connaissances entre les différents échelons de l'action publique, le Centre Alain Savary constitue ainsi un terrain significatif des modes actuels de gouvernement des politiques scolaires (Maroy, 2012; Van Zanten, 2013).

2. Circulaire du ministère de l'Éducation nationale, «Élaboration, pilotage et accompagnement des contrats de réussite des réseaux d'éducation prioritaire », 20 janvier 1999, Bulletin officiel de l'Éducation nationale, n² 4, 28 janvier 1999. 
Le présent article étudie dans cette perspective le positionnement du Centre Alain Savary, depuis sa création à Paris, en 1993, jusqu'à sa délocalisation-refondation à Lyon, en 2004. Ce positionnement n'est jamais monochrome : il varie suivant les contextes (en particulier politiques et institutionnels) et les activités - qui sont nombreuses, puisque, tout au long de la période, le Centre conduit simultanément des activités de recherche (financements d'équipes universitaires, contrats de recherche avec des organismes publics), de formation (organisation de stages pour des professionnels en ZEP) et de construction de ressources (publication d'ouvrages, bulletins et brochures, mise en ligne d'un site Web). Cependant, des dominantes ou inflexions - qui, secondaires relativement à cet entremêlement permanent des logiques de légitimation, sont placées ici au premier plan - peuvent toutefois être avancées à titre d'hypothèses. En se fondant sur les archives de ce centre et sur des entretiens avec certains de ses acteurs ${ }^{3}$, quatre périodes et positionnements successifs peuvent être distingués.

\section{UN CENTRE POUR LA SCIENCE ET POUR LA POLITIQUE (1992-1993)}

Les origines du Centre Alain Savary le situent à l'intersection de la politique de la ville (lorsqu'elle envisage l'école comme un levier pour agir dans les quartiers en difficulté), de la politique des ZEP (dont l'administration centrale considère qu'elle peut progresser par une meilleure formation des agents) et des travaux d'une équipe de recherche (qui trouve dans les ZEP un terrain pertinent pour enquêter sur les milieux populaires). Cette triple perspective, qui ne se juxtapose pas simplement à trois catégories d'acteurs, diversifie les objectifs du dispositif, tout à la fois pourvoyeur de ressources pour professionnels de ZEP, instrument d'orientation de la politique des ZEP et support pour la recherche scientifique. Le Centre Alain Savary connaît ainsi d'emblée un large répertoire d'activités dans lequel il puisera selon des dosages variables.

L'histoire commence en juillet 1990 lorsqu'un rapport sur la politique de la ville dans le département de Seine-Saint-Denis propose d'y créer un centre de ressources. L'idée est rapportée des États-Unis par la responsable d'une filière de la Caisse des dépôts et consignations (CDC) qui gère un parc immobilier sis dans

3. Cette étude est issue de travaux portant à titre principal sur la politique des ZEP (Bongrand, 2001, 2009), dans le cadre desquels ont été conduits des entretiens avec Patrick Bouveau et Bernard Charlot, présentés infra. Lors de leur consultation à Lyon en 2008-2009, les archives du Centre, notées ici ACAS, consistaient principalement en procès-verbaux du conseil scientifique. Les archives du bureau en charge des ZEP au sein de l'administration centrale du ministère de l'Éducation nationale au cours des années 1997-2004 (Archives nationales, versement 20050107) sont mobilisées ponctuellement. 
des territoires connaissant des difficultés analogues aux ZEP. Elle rencontre l'intérêt d'un professeur de sciences de l'éducation à l'université Paris VIII Saint-Denis, Bernard Charlot, en quête de partenariats pour développer l'équipe de recherche (ESCOL) qu'il y dirige. La CDC offre l'occasion de mener des enquêtes de grande ampleur; en octobre 1990, les chercheurs d'ESCOL pilotent ainsi un travail collectif d'évaluation des projets financés par la CDC dans les ZEP. Le centre de ressources Profession Banlieue, association hébergée par la Ville de Saint-Denis et financée par la CDC et la politique de la ville, est créé en 1993 (Charlot, 1994a). C'est dans ce contexte que la conseillère chargée des ZEP au sein du cabinet de Jack Lang, ministre de l'Éducation nationale, entreprend de développer la formation des professionnels en ZEP. L'idée de centre de ressources lui est suggérée, entre autres, par des militants socialistes liés à la politique de la ville et par l'Observatoire des zones prioritaires, une association créée en 1990 en soutien de la politique d'éducation prioritaire. Connaissant personnellement Bernard Charlot, la conseillère lui propose de réfléchir à la création du même genre de centre que pour la Seine-Saint-Denis. L'équipe ESCOL répond à cette sollicitation en commençant par constituer une banque de données d'actions éducatives dont elle distribue une version imprimée lors du colloque sur l'éducation prioritaire qui s'ouvre à Paris le 15 janvier 1993, dans les locaux de I'UNESCO4. Dans les semaines qui suivent, le «Centre de ressources sur les pratiques éducatives et sociales en milieux difficiles » est officiellement créés. Un an plus tard, il prend le nom d'Alain Savary, en mémoire du ministre de l'Éducation nationale ayant engagé la politique des ZEP en 1981.

Dans la continuité de ce processus, le texte fondateur du Centre l'inscrit pleinement dans les deux univers politique et scientifique. Sa vocation à la fois politique et savante apparait dans son statut, qui crée ce centre pour l'action publique « au sein de l'Institut National de Recherche Pédagogique (INRP) avec le concours de I'Université Paris VIII »; dans ses missions, puisqu'il doit « contribuer à la meilleure connaissance » des professions en milieux difficiles, mais également «à l'amélioration du travail des enseignants »; enfin dans son activité de promotion des pratiques novatrices en ZEP, à la fois « objets de développements, de formations, de recherches ». En cela, l'action du Centre mobilise les différents fondements de légitimité évoqués en introduction de cet article. Émanation de l'administration centrale pour inciter les acteurs de terrain à œuvrer en faveur

4. Centre de ressources sur les pratiques éducatives en milieux difficiles, Actions éducatives dans les zones d'éducation prioritaires et les quartiers en développement social urbain. Répertoire, Paris, 1993, conservé à la Bibliothèque nationale de France, cote 4-FW-10067.

5. Note «Centre de ressources sur les pratiques éducatives et sociales en milieux difficiles », 19 mars 1993, BOEN, $n^{\circ} 12,25$ mars 1993. 
de la politique des ZEP, le Centre s’adosse à la légitimité démocratique du ministère. En ne donnant pas d'orientation substantielle à l'action, en préférant faire connaître les « efforts et capacités d'invention » des acteurs de terrain, il insiste sur leur légitimité pragmatique. Mais en mobilisant des « partenaires universitaires associés », il suggère également une légitimation scientifique. Sur le papier, ces différentes manières de définir et de fonder le rôle du Centre apparaissent cohérentes : le Centre a vocation à participer à l'action publique d'éducation prioritaire autant qu'à mener des recherches. En pratique, elles balisent un espace en tension, où s'expriment des positionnements relativement contrastés.

\section{NI SCIENCE, NI POLITIQUE (1993-1996)}

Pour Patrick Bouveau, le premier responsable du Centre, les logiques politique, pédagogique et scientifique apparaissent indissociables, suivant une articulation qu'il a lui-même vécue tout au long de sa propre trajectoire professionnelle. "Fils d'ouvriers [devenu] instituteur », Patrick Bouveau devient "surnuméraire » en 1982 (i.e. maître sans classe, collaborant avec les autres enseignants) dans la ZEP de Clichy-Montfermeil, terrain d'enquêtes sociologiques (Bouveau, 1992). Nommé coordonnateur de ZEP à la rentrée 1985, il conduit des évaluations, puis se tourne vers l'Université. Militant politique et syndical, il est recruté en 1992 au cabinet de Bernard Tapie, éphémère ministre de la Ville. Dans ses travaux de sciences de l'éducation à Paris VIII, il s'interroge sur les rôles, marges de manœuvre et «stratégies » des différents professionnels qui exercent en ZEP. $C^{\prime}$ 'est donc à la fois sur un plan professionnel, politique et universitaire qu'il milite par et pour la politique des ZEP. Sa trajectoire lui permet de croire qu'il existe des pratiques plus efficaces que d'autres pour « réussir » en ZEP, que des recherches peuvent identifier de telles pratiques (voire y contribuer), qu'il est légitime de les faire connaître et qu'il appartient aux professionnels de terrain de s'en saisir. La socialisation à la recherche-action de Patrick Bouveau nourrit ainsi sa conviction que science et action publique sont compatibles.

Dans la présentation des activités du Centre, une perspective domine cependant, qui consiste à instrumenter l'ensemble des professionnels en rassemblant, en formalisant et en diffusant les contenus d'activité de certains d'entre eux. Si les logiques politiques et scientifiques apparaissent par endroits, le Centre doit avant tout « conscientiser » les professions de l'éducation prioritaire:

«Ses missions se résument en trois points:

- favoriser la connaissance et le développement d'initiatives spécifiques dans le cadre de certaines politiques territorialisées (zone d'éducation prioritaire, développement social des quartiers, contrat ville / enfant, contrat d'aménagement du temps de l'enfant...); 
- aider les opérations de formation soutenant ces initiatives (nationales, rectorales, locales); - assurer une liaison entre le développement de certaines pratiques éducatives et la recherche.

Le centre de ressources n'a donc pas pour vocation de créer un "organisme supplémentaire d'information" mais de réussir la mise en réseau de différents acteurs, formateurs et praticiens dans le cadre des stratégies éducatives territorialisées. » (INRP, 1993, p. 116-117)

Aussi le Centre bénéficie-t-il de contrats avec le Fonds d'Action Sociale pour les travailleurs immigrés et leurs familles (FAS), la CDC et la délégation interministérielle à la ville (DIV), d'un montant équivalent au double de la subvention de I'INRP'. II s'agit d'aider les acteurs à se connaître et à faire circuler les informations, qu'elles soient issues de productions scientifiques comme d'expériences pédagogiques. Les premières initiatives consistent à construire une base de données ( DIF-ACT ») et un bulletin des acteurs de ZEP ( $X, Y, Z E P »)$, de manière à promouvoir l'activité des agents intermédiaires qui, responsables, coordonnateurs ou enseignants, font exister l'échelon spécifique qu'est la « zone ». À partir d'avril 1993, la faible implication du cabinet du ministre François Bayrou dans l'éducation prioritaire ouvre au Centre un espace pour participer à l'animation de la politique des ZEP. Aux projets initiaux s'ajoutent, d'une part, l'évaluation de dispositifs locaux et, d'autre part, la mise en place de journées de formation: pour une politique déconcentrée, il s'agit d'outils d'administration typiques. Le Centre se rapproche ainsi du rôle d'une administration dans une logique de gouvernance.

Ce positionnement du Centre donne aux savoirs universitaires un statut secondaire et peu spécifique. L'INRP, qui travaille alors à son universitarisation (Prost, 1996), conçoit pourtant à cette époque ses « centres de ressources » comme des auxiliaires de la recherche en éducation, offrant des services de documentation ou de vulgarisation. Au contraire, l'intérêt prééminent pour la mise des acteurs en réseau situe le Centre Alain Savary à rebours de l'établissement public qui I'héberge. Après un an de fonctionnement, il n'est pas question de susciter des recherches purement universitaires. Si des chercheurs comme André Chambon ou Éliane Rogovas-Chauveau rattachent leurs travaux au Centre, les seules enquêtes menées en son nom sont des évaluations de dispositifs. Les savoirs que le Centre repère et manipule en priorité sont d'un autre ordre: non pas scientifiques, mais, plus largement, professionnels. II ne s'agit pas de les analyser et de publier les résultats de ces analyses, mais de recueillir leur contenu, le faire connaître, et le renvoyer d'où il vient - c'est-à-dire aux praticiens. On vise moins à transmettre des manières d'agir dont l'efficacité a pu être objectivement mesurée, qu'à mettre en place des lieux de discussion et de réflexion pour

6. «État des moyens engagés par le développement du Centre Alain Savary », document joint au dossier de préparation du comité scientifique du 30 janvier 1996, 5 pages, ACAS. 
mobiliser, « remotiver » des acteurs. Cette orientation duale se retrouve dans les critères de construction de la base de données DIF-ACT dès 1994 : un premier modèle recense les actions sans aucun filtre, en visant avant tout à susciter des contacts entre acteurs de ZEP; un second modèle, plus normatif, propose de sélectionner les actions selon des critères d'efficacité pédagogique, afin de mettre en valeur certaines pratiques. Le débat n'est pas tranché au moment où le changement de contexte politique bouleverse le positionnement du Centre.

\section{UNE POSITION DOUBLEMENT CRITIQUE (1997-1998)}

En 1997-1998, par contraste avec les années qui précèdent, le Centre est mis à l'épreuve à la fois en matière de savoirs universitaires et d'action publique, et il est amené à expliciter et défendre ses partis. Au fil des années en effet, les chercheurs de l'équipe ESCOL ont élaboré sur les ZEP un discours scientifique ferme et solidement étayé, éprouvé dans les enquêtes pour la CDC ou le FAS, conceptualisé dans des thèses de doctorat (Bouveau, 1994; Rochex, 1992) et des publications visant aussi bien acteurs que chercheurs en éducation (Charlot, Bautier \& Rochex, 1992). Son évaluation de l'action de la CDC pointe deux écueils complémentaires: I'absence de politique publique claire de la « grande banque », qui consent des financements sans les assortir d'objectif clair évaluable; la dilution des objectifs des ZEP au détriment de la lutte contre l'échec scolaire (Bouveau et al. , 1992, p. 52-53 ; Charlot, 1994b). Les chercheurs observent des acteurs soucieux de se montrer mobilisés plus que de faire réussir les élèves, des acteurs plus mobilisés pour le quartier que pour l'école. Ils assument le refus de juger leurs observations comme " effet positif »; a contrario, leur parti scientifique consiste à rappeler les spécificités de l'interaction scolaire. Le Centre affronte en 1997-1998 des situations qui mettent à l'épreuve la légitimité de ces thèses sur les plans universitaires et politiques.

Tenir cette ligne face à des interlocuteurs scientifiques ne va pas sans obstacle. Lorsque le poste de responsable du Centre Alain Savary est mis au concours, suivant des formes désormais universitaires, Patrick Bouveau, nommé responsable à la création du Centre, est largement pressenti. Réunie début février 1997, la commission de spécialistes de I'INRP recrute cependant une autre chercheuse. Lors de la réunion du conseil scientifique du Centre qui suit, chacun a son idée sur les bonnes et mauvaises raisons de ce recrutement ${ }^{7}$. La situation de porte-àfaux du Centre vis-à-vis de l'universitarisation de l'INRP d'une part, le « scolarocentrisme » de ses choix scientifiques d'autre part, trouvent ici à s'illustrer : ils

7. Comité scientifique, 25 mars 1997, ACAS. 
permettent d'interpréter cet épisode comme une conséquence du positionnement à contre-courant adopté jusqu'alors par le centre. À court terme, l'incident est toutefois contenu : Élisabeth Martin, agrégée de biologie, formée en troisième cycle universitaire par Bernard Charlot, prend officiellement la direction du Centre à la rentrée 1998. Elle en incarne la continuité scientifique.

Des épreuves proprement politiques bousculent plus durablement le positionnement du Centre. Celui-ci se trouve en effet directement associé à la « relance » de la politique des ZEP qui suit la nomination d'un gouvernement de gauche à l'issue des législatives de 1997. Constitué pour l'occasion, un comité de pilotage tient ses réunions au Centre - dont il a recruté une partie des membres du conseil scientifique - et travaille à produire une politique instruite par la recherche. Dans trois livrets confectionnés pour outiller l'action en ZEP, la perspective des travaux d'ESCOL prédomine, insistant sur le fait que les ZEP doivent être avant tout des lieux d'enseignement. Les « ressources » sont ainsi confectionnées dans une logique clairement top down, où des savoirs universitaires, empruntant des circuits bureaucratiques, viennent encadrer la réflexion des acteurs de terrain. Au même moment, les actions qui méritent d'être diffusées dans la banque de données DIF-ACT sont débattues en académie, lors de forums qui réunissent les professionnels de ZEP. Le Centre n’hésite pas à exprimer des critiques lorsque des prises de position de la ministre déléguée à l'Enseignement scolaire entrent en contradiction avec ce que démontrent les recherches. Dans la première édition du bulletin $X, Y, Z E P$, il prend par exemple ses distances avec les discours de Ségolène Royal au sujet des liens entre familles et école ${ }^{8}$.

Adossées à un fort investissement ministériel, les activités du Centre revêtent ainsi plus nettement une dimension politique. En participant au comité qui pilote la relance des ZEP, en sélectionnant des ressources, en proposant des orientations instruites par la recherche, en commentant les orientations politiques, le Centre semble désormais au moins autant promouvoir une politique publique singulière qu'apporter une contribution scientifique à l'action publique. Ce contexte de relance politique conduit ainsi les chercheurs du comité scientifique à positionner le Centre suivant des logiques plus directives. Mais il se retrouve alors en concurrence avec une administration centrale et une direction politique qui jouissent, elles, d'une légitimité légale-rationnelle. L'organisation de la relance suscite des conflits à l'issue desquels la ministre, d'une part, dénonce la distance des chercheurs à l'égard des acteurs dont ils prétendent

8. «Une lettre pour réfléchir sur les pratiques en ZEP », La lettre de l'éducation, n²21, 19 janvier 1998, p. 2. 
rendre compte, et, d'autre part, met à distance leur prétention à orienter la politique (Bongrand, 2001). Cet épisode impose au Centre un nouveau positionnement, qui s'esquisse au fil des années 1999-2004.

\section{SCIENCE (D'UNE PART), POLITIQUE (DE L'AUTRE) (1999-2004)}

Mis en quarantaine par le ministère à partir de la rentrée 1998, le Centre s'interroge: "Cette année, nous vivons un "état de disgrâce" de la part du cabinet qui bouscule notre légitimité, voire notre existence [...]. Comment se repositionner? $»^{9}$. La réponse le rapproche du champ scientifique, où il jouit d'une marge de manœuvre plus large qu'au sein des politiques scolaires. Les sources de financement n'y sont pas les mêmes, et le terrain y apparaît moins disputé par l'administration centrale - où la direction compétente en matière de recherche, désormais intitulée Direction de la programmation et du développement, se trouve alors malmenée. Le Centre élabore un « appel à coopération » inédit au cours de I'hiver 1998-1999. Visant non pas des objectifs politiques, mais des questions scientifiques, il conduit à financer et coordonner six recherches. Le rôle des enseignants associés au Centre est revu dans une perspective de production de recherches, consistant non plus à alimenter la banque de données DIF-ACT, mais à s'insérer dans des équipes universitaires. À la rentrée 2000, la responsabilité du Centre est confiée à une universitaire recrutée par voie de détachement, Martine Kherroubi, issue de l'équipe de sociologie de l'éducation de Paris V, spécialiste des pratiques et professions enseignantes. Dans la mesure où la nouvelle directrice appartient à une équipe financée suite à l'appel à coopération, sa nomination revient à faire poursuivre la recherche à l'intérieur même du Centre. En plus de poursuivre ses propres travaux, Martine Kherroubi co-écrit, avec un membre du comité scientifique du Centre, une vaste note de synthèse sur la recherche sur les ZEP (Kherroubi \& Rochex, 2002, 2004), qui entend baliser un champ académique avant d'en tirer des « ressources». Au tournant des années 1990 à 2000, le balancier se déplace ainsi vers la figure d'un centre pour la recherche en même temps que centre de recherche.

Cette scientifisation du Centre progresse en raison d'un différend avec l'administration centrale du ministère, puis d'une réorganisation de la production et de l'élaboration de « ressources ». À partir de 1998 en effet, l'administration centrale fait naître un bureau, le « Desco B5 », qui reprend en main la politique des ZEP par la voie d'activités analogues à celles amorcées ou envisagées jusqu'alors

9. Comité scientifique, 16 octobre 1998, ACAS. 
par le Centre Alain Savary. Dès la rentrée 1998, le B5 organise par exemple une université d'été pour accompagner les ZEP dans l'élaboration de leurs « contrats de réussite » - opérant une mise au ban du Centre si manifeste, que certains de ses membres songent à riposter par une formation concurrente ${ }^{10}$. Le développement contemporain de sites Web dans l'administration attise cette rivalité: alors que le Centre administrait jusqu'alors le principal site officiel au sujet des ZEP, le B5 inaugure un nouveau site au printemps 1999. Enfin, plutôt que de le « remettre au pas $»^{11}$, l'administration ignore le Centre Alain Savary et son comité scientifique au moment de préparer une nouvelle édition des Assises de l'éducation prioritaire à Strasbourg en juin 2000 - assises qu'ils avaient pourtant organisées ensemble en 1998.

Ce n'est qu'au fil des années 2000-2004 que le Centre renoue, discrètement, avec la logique de ses débuts. Lorsque la direction politique se fait moins présente en matière de ZEP sous le ministère Lang (2000-2002), le Centre peut entreprendre de travailler à nouveau avec le B5 et de relancer progressivement ses activités de production de ressources. La parution du bulletin $X, Y, Z E P$ reprend - quoique de manière irrégulière: un à trois des quatre numéros annuels annoncés paraissent en 1998-1999 et 2004-2005. Le Centre élabore une lettre d'information électronique pour les coordonnateurs de ZEP (lettre " Réseau-coordo »), à raison de quatre à cinq numéros annuels au cours des trois années scolaires 2002-2005. En 2003-2004 enfin, Dominique Millet, agrégé de philosophie, est nommé co-directeur du Centre par le ministère. Entre-temps, la partie recherche s'est comme autonomisée.

C'est sur ce registre dichotomique que les relations du Centre avec la DESCO s'apaisent. Alors que l'inquiétude se manifeste encore début 2000², le Centre se repositionne comme centre d'expertise sur les recherches en ZEP et est associé en 2002 sur ce registre aux célébrations des vingt ans des ZEP. Martine Kherroubi met en avant cette même fonction d'expertise dans le texte qu'elle propose au B5 d'insérer dans la circulaire destinée aux ZEP pour la rentrée 2003 : le Centre s'y distingue, parmi les différents acteurs qui élaborent et font circuler des ressources, par sa légitimité proprement scientifique ${ }^{13}$. Cette proposition montre

10. Notes d'Élisabeth Martin relatives au comité scientifique du 8 novembre 1998, ACAS.

11. Suivant les termes d'un court filet paru, sans commentaire, dans Libération, 25 mai 1999, p. 18. Voir également «Climat tendu à la DPD et inquiétudes au Centre Alain Savary », La lettre de l'éducation, $n^{\circ} 265,29$ mars 1999, p. 1.

12. En janvier 2000, la responsable du Centre s'interroge : «Faut-il reprendre contact avec le ministère? Le Centre doit avant tout garder son indépendance et avoir son plan de travail propre », Comité scientifique, 7 janvier 2000, ACAS.

13. Courrier électronique de Martine Kherroubi au ministère, 13 février 2003, versé au fonds 20050107, Archives nationales, site de Pierrefitte-sur-Seine. 
combien l'espace institutionnel dans lequel s'insère le Centre a changé. Envisagé rétrospectivement, le Centre fait en 1993 figure de pionnier : sans concurrent, il n'a pas besoin d'expliciter et de justifier un positionnement « scientifique » ou « politique » pour agir en matière de politique de l'éducation prioritaire. Dix ans plus tard, après avoir éprouvé la difficulté à tenir ensemble ces différentes positions, il se présente comme l'une des composantes d'un réseau de centres de ressources, où il se distingue, précisément, par ses liens avec la recherche.

Ces moments successifs de l'histoire du Centre Alain Savary montrent que «l'expertise », entendue comme manière d'associer des chercheurs à une action publique, peut revêtir des formes diverses et précaires, sensibles aux contextes politico-institutionnels. Dans le champ d'action publique déconcentrée et instrumentée que constitue l'éducation prioritaire, mobiliser la science au bénéfice de la circulation de ressources ne suscite qu'un accord de principe : en pratique, à l'échelle d'une décennie, chercheurs comme politiques ne trouvent pas d'arrangement durable. Ces péripéties montrent la difficulté à légitimer une politique fondée sur la circulation d'instruments : à moins de tout faire circuler, il faut un principe de régulation; or si le regard scientifique est volontiers sollicité pour réguler cette circulation, les jugements scientifiques y endossent une portée qui redouble ou heurte, de facto, la régulation proprement politique. Pour les acteurs tout autant que pour les analystes, ces nouvelles formes d'action publique soulèvent ainsi la question de savoir qui, dans un tel contexte, gouverne (Favre, 2003). ${ }^{14}$

Philippe Bongrand

École, Mutations, Apprentissages (ÉMA-EA 4507) Université de Cergy-Pontoise

14. Merci au Centre Alain Savary d'avoir permis la consultation de ses archives, ainsi qu'à Martine Kherroubi, aux évaluateurs anonymes et aux co-directeurs de ce dossier pour leur lecture critique d'une version antérieure de ce texte, qui ne saurait en aucun cas les engager. 


\section{LA LÉGITIMITÉ PRÉCAIRE DE L'EXPERTISE}

\section{Bibliographie}

Bongrand P. (2001). La réflexivité de l'action publique. Savoirs et producteurs de savoirs dans la relance des zones d'éducation prioritaires (1997-1998), mémoire de DEA en science politique, Université Paris 1 Panthéon-Sorbonne.

Bongrand P. (2009). La scolarisation des mours. Socio-histoire de deux politiques de scolarisation, en France, depuis la Libération, thèse de doctorat en science politique, Université de Picardie Jules Verne.

Bouveau P. (1992). D’Auteuil aux Bosquets. L'essence d'une profession, Perspectives documentaires en éducation, 25, 53-61.

Bouveau P. (1994). Des stratégies éducatives territorialisées aux changements: place et rôle des enseignants dans les ZEP urbaines, thèse de doctorat en sciences de l'éducation, SaintDenis : Université Paris 8.

Bouveau P. et al. (1992). Le soutien aux politiques éducatives dans les ZEP DSQ (1989-1992). Évaluation de l'impact de la Caisse des Dépôts et Consignations (convention tripartite MENDIV-CDC), association ANALISE.

Charlot B. (1994a). Le partenariat entre I'Université et les chefs de projet : la création du centre de ressources Profession Banlieue (pp. 287-384). In É. Bautier et al., Les professionnels et la professionnalisation en banlieue. Rapport remis au ministère de l'Enseignement supérieur et de la Recherche et au ministère de l'Équipement, des Transports et du Tourisme. Charlot B. (1994b). «Ce qui se pense » dans les zones d'éducation prioritaires. Analyse des demandes de financement. In B. Charlot (coord.), L'école et le territoire: nouveaux espaces, nouveaux enjeux (pp. 121-142). Paris: Armand Colin.

Charlot B., Bautier É., Rochex J.-Y. (1992). École et savoirs dans les banlieues... et ailleurs. Paris: Armand Colin.

Delmas C. (2011). Sociologie politique de l'expertise. Paris : La Découverte.

Dutercq Y. (2005) (dir.). Les régulations des politiques d'éducation. Rennes: Presses universitaires de Rennes.

Favre P. (2003). Qui gouverne quand personne ne gouverne? In P. Favre, J. Hayward, Y. Schemeil (dir.). Être gouverné. Études en l'honneur de Jean Leca (pp. 259-271). Paris : Presses de Sciences Po.

INRP (1993). L'Institut national de recherche pédagogique. Recherches, ressources, structures. 1993-1994. Paris: INRP.

Kherroubi M., Rochex J.-Y. (2002). La recherche en éducation et les ZEP en France. 1. Politique ZEP, objets, postures et orientations de recherche, Revue française de pédagogie, 140, 103-132.

Kherroubi M., Rochex J.-Y. (2004). La recherche en éducation et les ZEP en France. 2. Apprentissages et exercice professionnel en ZEP : résultats, analyses, interprétations, Revue française de pédagogie, 146, 115-190.

Lascoumes P., Le Galès P. (2005) (dir.). Gouverner par les instruments. Paris : Presses de Sciences Po.

Maroy C. (2012) (Ed.), Réguler les systèmes scolaires par les connaissances : instruments, usages, effets (numéro thématique), Recherches sociologiques et anthropologiques, 43, 2. 
Pasquier R., Simoulin V., Weisbein J. (2007) (dir.). La gouvernance territoriale. Pratiques, discours et théories. Paris: LGDJ.

Ravon B. (2000). L'« échec scolaire». Socio-histoire d'un problème public. Paris : In Press.

Rochex J.-Y. (1992). Entre activité et subjectivité : le sens de l'expérience scolaire, thèse de doctorat en sciences de l'éducation, Saint-Denis : Université Paris 8.

Prost A. (1996), Regard. In INRP 1976-1996. 20 ans de recherche en éducation (pp. 11-27). Paris : INRP.

Van Zanten A. (2011). Les politiques d'éducation. Paris: PUF.

Van Zanten A. (2013) (Ed.). Connaissances et politiques d'éducation : quelles interactions? (Numéro thématique), Revue française de pédagogie, n 182. 